\title{
Doenças lisossômicas de depósito em pacientes com possíveis diagnóstico de erros inatos do metabolismo
}

\section{Lysosomic diseases of deposit in patients with possible Inborn Errors of Metabolismdiagnosis}

\author{
Cíntia Costa MedeirosMartins ${ }^{1}$ \\ Thomas WerlandLima ${ }^{2}$ \\ Jaqueline Cé ${ }^{3}$ \\ Janice Carneiro Coelho ${ }^{4}$ \\ *Alexandre Silvade Mello ${ }^{5}$
}

\section{RESUMO}

As Doenças Lisossômicas de Depósito (DLDs) são distúrbios genéticos, clinicamente heterogêneos, causados principalmente por defeitos em genes que codificam enzimas lisossômicas que degradam macromoléculas. Este estudo tem o objetivo de verificar o índice de DLDs na coleta de sanguede 4986 pacientes, com possíveis diagnósticos de uma destas doenças de um laboratório de referêncialocalizado no Sul do Brasil, com amostras de todos os Estados do País.Trata-se de um estudo quantitativo, transversal de caráter descritivo e exploratório, com dados secundários coletados pela pesquisa realizada no Laboratório de Bioquímica da Universidade Federal do Rio Grande do Sul- UFRGS, entre os anos de 2010 a 2015, para a pesquisa, foi utilizado um banco de dados que já estavam arquivados em uma planilha de Excel. Nesse estudo, avaliamos protocolos para o diagnóstico confirmatório de casos. Inicialmente, revisamoscasos triados positivos para Doença de Fabry, Doença de Gaucher, Doença de Pompe, Mucopolissacaridose eNiemann-Pick. Esse estudo discute também as limitações das análises enzimáticas e moleculares na confirmação do diagnóstico, os desafios que podem ser encontrados durante a interpretação dos resultados e a necessidade de um protocolo abrangente para a investigação precisa de casos em suspeita, identificados por estudos para DLDs. Esse estudo representa uma base para avaliações mais abrangentes, em vista de uma possível implementação diagnóstica em grande escala.

\section{PALAVRAS-CHAVE}

Doenças lisossômicas de depósito; Erros Inatos do Metabolismo; Doenças Raras.

\footnotetext{
${ }^{1}$ Doutoranda do Programa de Pós-Graduação em Ciências da Reabilitação da Universidade Federal de Ciências da Saúde de Porto Alegre (UFCSPA).

${ }^{2}$ Graduando em Biomedicina pelo Centro Universitário Metodista (IPA).

${ }^{3}$ Doutora do Programa de Pós-Graduação em Ciências Biológicas: Bioquímica.

${ }^{4}$ Professora Doutora do Departamento de Bioquímica da Universidade Federal do Rio Grande do Sul (UFRGS).

${ }^{5}$ Professor Doutor do Programa de Pós-Graduação em Biociências e Reabilitação do Centro Universitário Metodista (IPA).
} 


\section{ABSTRAT}

Lysosomal Deposit Diseases (DLDs) are genetic disorders, clinically heterogeneous, caused mainly by defects in genes that encode lysosomal enzymes that degrade macromolecules. This study aims to verify the index of DLDs in the blood collection of 4986 patients, with possible diagnoses of one of these diseases in a reference laboratory located in southern Brazil, with samples from all states in the country. This is a quantitative, cross-sectional study of a descriptive and exploratory character, with secondary data collected by the research carried out at the Biochemistry Laboratory of the Federal University of Rio Grande do Sul- UFRGS, between the years 2010 to 2015, for research, a database that was already filed in an Excel spreadsheet was used. In this study, we evaluated protocols for confirmatory diagnosis of cases. Initially, we reviewed positive screened cases for Fabry disease, Gaucher disease, Pompe disease, Mucopolysaccharidosis and Niemann-Pick. This study also discusses the limitations of enzymatic and molecular analyzes in confirming the diagnosis, the challenges that can be encountered when interpreting the results and the need for a comprehensive protocol for the accurate investigation of suspected cases, identified by studies for DLDs. This study represents a basis for more comprehensive assessments, in view of a possible large-scale diagnostic implementation.

\section{KEYWORDS}

Lysosomal Deposit Diseases; Inborn errors of metabolism; Rare diseases. 


\section{INTRODUÇÃO}

Os Erros Inatos do Metabolismo (EIMs) são doenças hereditárias raras, transmitidas de forma autossômica recessiva, determinadas geneticamente causadas por um defeito específico, geralmente enzimático, que leva ao bloqueio de uma determinada via metabólica. Esse bloqueio tem como consequência o acúmulo do substrato da enzima deficiente, ocasionando a diminuição do produto da reação ou o desvio do substrato para uma via metabólica alternativa. A prevalência de EIMs, apresenta uma incidência cumulativa estimada em 1:1.000 recém-nascidos vivos (EMENSet al., 2019; ZHOU et al., 2018; KARAM et al., 2001).

Entre essas patologiasestá aDoença de Gaucher, que é uma das enfermidades portadoras de erros enzimáticos, tendo em vista a deficiência no depósito lisossomal, caracterizada pelo acúmulo de glucosilceramida nas células do sistema macrófago/monócito, em decorrência da deficiência hereditária da enzima lisossomal $\beta$-glicosidase ácida.Em 1882, o médico francês Phillippe Charles Ernest Gaucher descreveu a doença, pela primeira vez, em uma mulher adulta, cujo fígado e baço estavam aumentados (PRADHAN et al., 2017).

Nesse contexto de herança genética, pode-se citar, também, a Doença de Pompe,uma patologia caracterizada por uma disfunção no armazenamento lisossômico, manifestada por umdéficitna enzima alfa ácidoglicosidase, que ocasiona um acúmulo de glicogênio dentro do lisossomo. A causa de depósito de glicogênio é a deficiência da atividade de uma enzima lisossômica, a alfa 1,4 glicosidade ou maltase ácida, sendo uma doença transmitida de forma autossômica recessiva. A incidência estimada é de 1 em cada 40000 nascidos, originando danos e disfunções orgânicas no tecido muscular, e, assim, dificultando a respiração, locomoção, fala e audição. A doença pode-se apresentar em três formas: a infantil, a juvenil e a adulta (ZHOU et al., 2018; BERMAN et al.,1974;).

Somado a esses erros inatos do metabolismo, acrescenta-se a alteração do estado de saúde denominado comoDoença de Mucopolissacarídeos (MPS),uma doença rara transmitida de maneira autossômica recessiva causada por deficiência enzimática. É considerado como sendo o maior grupo de doenças genéticas do metabolismo, pois a deficiência de enzimas degrada os GAGs (glicosaminoglicanos). Além disso, os acúmulos de GAGs geram a doença de MPS, que pertencem a um subgrupo das Doenças Lisossômicas de Depósito (DLD). Essa patologia foi descrita pela primeira vez por Berman e colaboradores em 1974 e foi caracterizada por manifestar em seus portadores:deficiências intelectuais, turvação da córnea, degeneração da retina e acloridia, podendo levar à hipergastrinemia(FERREIRA, 2017; BERMAN et al.,1974).

Existem diferentes tipos de MPS, cada uma causada por um diferente tipo de deficiência enzimática. Entre elas está a Doença de Fabry, descrita em 1898 por dois dermatologistas, Johannes Fabry, na Alemanha, e William Anderson, na Inglaterra (ZHOU et al., 2018).

A doença de Fabry é uma patologia de herança genética ligada ao cromossomo X. Ela provoca uma atividade insuficiente da enzima lisossômica Alfa galactosidase-A ( $\alpha$-gal A) eacometecom frequência,disfunções ou danos celulares frequentemente no tecido renal, cardíaco, pele e córneas. As mutações no gene GLA localizado no cromossomo Xq22são responsáveis por essa deficiência, sendo descritas mais de 600 mutações nesse gene até o momento. As mutações podem ser missense, nossense e inserção ou seleção de único aminoácido. As mutações são privados em sua maioria, ou seja, particulares daquela família. A incidência da doença, na população geral, foi estimada em 1:117000, embora possa estar subestimada.

O quadro clínico é variável e progressivo, com acometimento de múltiplos órgãos. Geralmente tem o seu início na infância ou adolescência, com crises periódicas de acroparestesia, aparecimento de lesões cutâneas vasculares, hipohidrose e distúrbios gastrointestinais (BERNARDES et al., 2020).

Já a Doença de Niemann-Pick é uma doença hereditária, de caráter autossômico recessivo e rara, caracterizada pela deficiência de esfingomielinase ácida, que determina o acúmulo de esfingomielina, principalmente nos tecidos do sistema reticuloendotelial. Em 1934, Ernst Klenk identificou o lípido armazenado como esfingomielina. Em 1961, Crocker propôs classificar a doença em tipos A,B e C. Em 1966 que Roscoe Brady encontrou uma deficiência de ácido esfigomielinase em pacientes afetados com doença de Niemann-Pickdo tipo A e B, mas não para o tipo C (SABATINI, 2014; BRADY, 1966; CROCKER, 1961). 
Os diferentes tipos de doença de Nieman-Pick formam diferentes quadros clínicos. A variante mais comum e grave é o tipo A, formaneuronopática aguda. Os fatores que determinam a doença de Niemann-Pick são manifestados nos indivíduos afetados, em grande parte, por aqueles que possuem sintomas em decorrência de acúmulo de macrófagos repletos de lipídios e vacuolizados em vários órgãos, como fígado, baço, medula óssea, pulmão e sistema nervoso central. A doença é classificada em seis subtipos, de A à F (VANIER et al.,1993).

No Brasil, o embrião para a discussão de tais políticas foi a criaçãodo grupo de trabalho para a elaboração de uma proposta para Política Nacional de Atenção à Genética Clínica no SUS, durante a década de 2000. No entanto, após algumas tentativas, viu-se que essa abordagem não contemplava as necessidades assistenciais específicas de muitas doenças, uma vez que focava apenas nas doenças de origem genética. Em 2014, a partir da demanda da sociedade, através de organizações não-governamentais e instituições de pesquisa, que discutiam o assunto há alguns anos, o Ministério da Saúde publicou, no Diário Oficial da União, a Portaria que cria a Política Nacional de Atenção Integral às Pessoas com Doenças Raras, incorporando exames e credenciando instituições para atendimentos de pacientes com essas condições (WASSERSTEIN, 2015).

À medida que países emergentes economicamente, como o Brasil, conseguem sanar algumas causas de mortalidade, como a desnutrição e outras doenças comuns, as doenças raras passam a receber destaque no cenário de saúde pública. 0 rápido avanço científico e tecnológico tem permitido intervir em diversas condições de saúde, incluindo as doenças raras. Os avanços na genômica acarretam a definição mais precisa das doenças e, consequentemente, o desenvolvimento de tratamentos inovadores (MAHER\& HAFFNER, 2006).

Diante da necessidade de tratamentos para doenças raras, nos últimos dez anos, parcerias público-privadas entre instituições acadêmicas, companhias farmacêuticas, associações e Governo, têm sido bem-sucedidas no exterior. As legislações americana e europeia para medicamentos órfãos têm auxiliado a transformar as medicações para tais doenças em rentáveis para trazê-las ao mercado (KHARROUBI et al., 2018).

\section{METODOLOGIA}

\section{ASPECTOS ÉTICOS}

Levando em consideração o exposto acima, este estudo teve como objetivo estabelecer a frequência de Doenças Lisossômicas de Depósito em uma amostra de pacientes com suspeita destas doenças num banco de dados do Laboratório de Doenças Lisossômicas de Depósito no Departamento de Bioquímica da Universidade Federal do Rio Grande do Sul- UFRGS e analisar a metodologia usada neste estudo.

\section{DESENHO, LOCAL DO ESTUDO E PERÍODO}

Trata-se de um estudo quantitativo, transversal de caráter descritivo e exploratório, com dados secundários coletados pela pesquisa realizada no Laboratório de Doenças Lisossômicas de Depósito no Departamento de Bioquímica da Universidade Federal do Rio Grande do Sul- UFRGS, entre os anos de 2010 a 2015, para a pesquisa foi utilizado um banco de dados que já estavam arquivados em uma planilha de Excel.A população que participou do estudo foram 4986 pacientes de ambos os sexos, sem delimitação de idade, do Brasil inteiro.

Foram coletadas amostras de leucócitos, plasma sanguíneo desses pacientes. As amostras foram encaminhadas para o laboratório da mesma instituição, onde foram processadas as amostras e arquivadas, e com a emissão de respectivos exames arquivados fisicamente e virtualmente em uma planilha de Excel.Também foram utilizadas outras variáveis para a caracterização das amostras como: sexo, estado, cidade, número de pacientes por cidade e estado diagnosticados com a doença.Foram excluídos aqueles pacientes que estavam com diagnóstico duvidoso e pacientes com diagnóstico normal.

\section{RESULTADOS}

Entre os 4986 pacientes de 2010 a 2015 estudados de acordo com a tabela 1, 2627 (58\%) do sexo masculino e 2359 (42\%) do feminino, a média de idade apresentada foi de $0 \pm 60$ anos. Foram diagnosticadas doenças que fazem parte do grupo deEIMsem 282 amostras conforme as tabelas de $1 \mathrm{a} 4$. 
Tabela 1- Descrição de indivíduos com a Doença Lisossômica de Depósito

\begin{tabular}{c|c}
\hline Doenças & Indivíduos com DLD \\
\hline Fabry & 39 \\
Gaucher & 169 \\
MPS I & 9 \\
Pompe & 34 \\
MPS VI & 9 \\
NPB & 2 \\
NP & 20 \\
\hline Total & $\mathbf{2 8 2}$ \\
\hline
\end{tabular}

Legenda: MPS I: Mucopolissacaridose tipo 1; MPS VI: Mucopolissacaridose tipo 6; NPB: Niemann-Pick tipo B; NP: Niemann- Pick
Tabela 2 - Descrição da prevalência de gêneros por doença

\begin{tabular}{cc}
\hline Doenças & Sexo \\
\hline Fabry & M:29/ F:10 \\
Gaucher & M:73/F:80 \\
MPS I & M:15/F:19 \\
Pompe & M:6/ F:5 \\
MPS VI & M: 1/ F:1 \\
NPB & M:11/ F:9 \\
(Niemann- Pick) & M:140/ F:128
\end{tabular}

Legenda: M: Masculino; F: Feminino.

Legenda: MPS I: Mucopolissacaridose tipo 1; MPS VI: Mucopolissacaridose tipo 6; NPB: Niemann- Pick tipo B; NP: NiemannPick.

Tabela 3 - Tabela descritiva dos Estados com maiores ocorrências de DLD

\begin{tabular}{ccccccccc}
\hline Estado & Fabry & Gaucher & MPS I & Pompe & MPS VI & NPB & NPC & NP \\
\hline SP & 39 & 24 & 4 & - & 9 & - & 6 & - \\
RJ & - & - & - & 9 & - & - & - & - \\
AL & - & - & - & - & - & 1 & - & - \\
DF & - & - & - & - & - & 1 & - & - \\
PE & - & - & - & - & - & - & - & 4 \\
\hline
\end{tabular}

Legenda: SP: São Paulo; RJ: Rio de Janeiro; AL: Alagoas; DF: Distrito Federal; PE: Pernambuco.

Legenda: MPS I: Mucopolissacaridose tipo 1; MPS VI: Mucopolissacaridose tipo 6; NPB: Niemann- Pick tipo B; NP: Niemann- Pick.

Tabela 4 - Descrição das cidades com maior ocorrência das doenças

\begin{tabular}{ccccccccc}
\hline Cidades & Fabry & Gaucher & MPS I & Pompe & MPS VI & NPB & NPC & NP \\
\hline Ribeirão Preto (SP) & 39 & 8 & 2 & 4 & - & - & 5 & 3 \\
São Paulo (SP) & - & 5 & - & - & 4 & 1 & - & \\
Salvador (BA) & - & 13 & - & - & - & - & - & - \\
Goiânia (GO) & - & 5 & - & - & - & - & - & - \\
São Luís (CE) & - & 9 & - & - & - & - & - & 4 \\
Belo Horizonte (MG) & - & 7 & - & - & - & - & - & - \\
& & & - & - & & & & \\
Curitiba (PR) & - & 8 & - & - & - & - & - & - \\
Rio de Janeiro (RJ) & - & 13 & - & - & - & - & - & - \\
Aracajú (SE) & - & 9 & - & - & - & - & - & - \\
Recife (PE) & - & - & - & - & - & - & - & 4 \\
Manaus (AM) & - & 5 & - & - & - & - & - & - \\
Maceió (AL) & - & - & - & - & - & 1 & - & - \\
\hline
\end{tabular}

Legenda: SP: São Paulo; AM: Amazonas; BA: Bahia; GO: Goiás; CE: Ceará; MG: Minas Gerais; PR: Paraná; RJ: Rio de Janeiro; SE: Sergipe; PE: Pernambuco; SP: São Paulo; AL: Alagoas. 


\section{DISCUSSÃO}

Do total de indivíduos analisados e diagnosticados com DLDs neste estudo, a Doença de Gaucher tem um total de $40 \%$ da amostra, tendo uma prevalência maior no Estado de São Paulo, onde o maior número de portadoras da doença é do sexo feminino. Segundo Gaucher (2007), a Doença de Gaucher é uma doença com incidência de 1:40.000 nos Estados Unidos, correspondendo uma maior prevalecia nos judeus Ashkenazi (1:400 a 1:800). Em 2007, dos 4936 pacientes cadastrados no InternationalCollaborativeGaucherGroup(ICGG)乞o maior banco de dados do mundo de pacientes portadores de Doença de Gaucher, os destaques da epidemiológicos da Doença de Gaucher, são os EUA (1780 pacientes, 36\%), Israel (683 pacientes, 13,8\%), Brasil (496 pacientes, 10\%) e Reino Unido (253 pacientes, 4,7\%) (SOBREIRA\&BRUNIERA,2008).

Assim, de acordo com o mesmo, grupo de pesquisadores (Gaucher, 2010),dos 5915 pacientes cadastrados no ICGG, 561 são do Brasil, entre esses pacientes 3,5\% são do Ceará, Estado com 8,2 milhões de habitantes e35\% pacientes deriva de Tabuleiro do Norte, um pequeno município de 28.000 habitantes, no Estado do Ceará,localizado na região Nordeste do Brasil. A população de doentes de Gaucher do Estado de São Paulo, da mesma forma como no mundo inteiro, é constituída na sua grande maioria por pacientes com a variante não-neuropática ou do tipo 1 (FERREIRA et al., 2011).

Na população estudada em São Paulo, houve predomínio no sexo feminino, embora não haja explicação pela natureza da herança genética envolvida.A classificação étnica dos pacientes brasileiros é complexa, devido às diferentes miscigenações ocorridas nas famílias desde os tempos da colonização. Em São Paulo, a maioria dos pacientes é composta por não-brancos. Apesar da doença do tipo 1 ser muito frequente entre os judeus Ashkenaznesta amostra estudada nenhum paciente apresentava esta origem, por isso esta classificação étnica não foi utilizada. A hepatomegalia e ou esplenomegalia foi quase universal, e pelo menos metade apresentava anemia e/ou trombocitopenia e dor óssea. Estas são as manifestações que mais frequentemente levam ao diagnóstico de Doença de Gaucher 1, de acordo com a literatura (BERNSTEINet al., 2019;SOBREIRA et al., 2007).
No entanto, a Associação de Pacientes com Doença de Gaucher informou que em 2011, havia 614 pacientes com Doença de Gaucherem todo Brasil. Com superioridade na Região Sudeste (57\%) dos pacientes. Em São Paulo (178 pacientes), Minas Gerais (84 pacientes) e Rio de Janeiro (76 pacientes) são os Estados brasileiros com maior número de pacientes com DG (CHAVES et al.,2018).

A Doença de Gaucher tem origem judaica, foi descoberta há dez anos por um médico em Tabuleiro do Norte, no Vale do Jaguaribe. Até hoje, a cidade continua no topo do ranking de maior incidência no país. Em Tabuleiro do Norte, a incidência da Doença de Gaucher é 25 vezes maior do que a média nos outros lugares do planeta. A alta concentração de casos na cidade tem um fato histórico e remete ao passado, quando uma família de imigrantes portugueses chegou na região Jaguaribana, em 1690, há 326 anos. Dez anos depois, a maior incidência no Brasil ainda é em Tabuleiro do Norte. Estudos recentes indicam que é possível realizar o tratamento e o aconselhamento genético para a população tabuleirense (BREIGEIRON et al.,2018).

A Doença de Gaucher, a mais comum das doenças de depósito lisossômico, é um distúrbio autossômico recessivo devido à deficiência de glucocerebrosidase ( $\beta$-glucosidase ácida), que converte o glucocerebrósidoa ceramida e glicose. Como consequência deste defeito, o glucocerebrósido (GL1), umno catabolismo de globóides e gangliosídeos, se acumula no sistema reticuloendotelial. A herança é autossômica recessiva, sua incidência global é desconhecida, porém se estima que seja de 1:7.000 ou 1:10.000 Judeus Ashkenazim,mas não existe uma prevalência sugerindo que essas doenças apareceram no Brasil por contada imigraçãojudaica na época da Segunda Guerra Mundial (PASTORES, et al; 2018; A., ZIMRAN et al, 2019;MOZAFARI, $\mathrm{H}$ et al, 2020)

Um levantamento feito na Austrália registrou uma frequência de 1: 57.000. Um estudo semelhante, feito na Holanda, relatou 1: 100.000.Nessa população, a prevalência da doença é de 1:855 e a frequência estimada de portadores é de 1:18. Mais de 90\% das mutações em Judeus Ashkenazi podem ser detectados pelo rastreamento das mutações mais comuns, e aproximadamente $50 \%$ das mutações em populações não judaicas podem ser detectadas dessa maneira. No entanto, a porcentagem exata depende sobre o número de mutações co- 
muns sendo rastreadas e sobre a etnia da população. A prevalência da doença de Gaucher neuropática (tipos 2 e 3) varia entre os grupos étnicos, mas parece ser maior entre indivíduos que não sejam de raça branca (COSTA et al., 2018; FERREIRA, 2017; BARNESS, 1987).

Se ambos os pais forem portadores, a criança terá chance de 1:4 (ou 25\%) de ter a Doença de Gaucher, chance de 1:2 (ou 50\%) de ser portadora sem apresentar a doença e chance de 1:4 (ou 25\%) de não ter a doença nem ser portadora (Matos et al., 2018; Barness, 1987).Com Base dos dados deste estudo, a Doença de Fabry é mais prevalente em São Paulo $(13,8 \%)$, com um número maior no sexo masculino.

A Doença de Fabry foi descrita pela primeira vez por dois dermatologistas que trabalhavam em diferentes locais: um na Inglaterra e o outro na Alemanha, no ano de 1898. A partir dos dados históricos, é interessante notar que a diferença da descrição dos primeiros casos da doença é de meses: Johannes Fabry teria examinado seu primeiro paciente em abril de 1897, e William Anderson em dezembro de 1897. Por isso, embora mais comumente chamada de Doença de Fabry, também é conhecida como doença de Anderson-Fabry. Os primeiros pacientes com Doença de Fabry foram descritos pelo dermatologista alemão Johannes, em $\underline{1898}$. Fabry e o dermatologista inglês William Anderson realizaram suas pesquisas de forma independente. A Doença de Fabry foi trazida pelas imigrações de avós maternos que vieram da Alemanha, através de imigração em um barco para o Brasil (CASSIANO, 2017).

A Doença de Fabry já foi descrita em diversas etnias, sem diferenças significativas entre elas até o presente momento. A prevalência estimada da doença é de aproximadamente 1:40.000 indivíduos do sexo masculino. Estudos recentes em recém-nascidos encontraram uma incidência elevada, variando de 1:3.100 a 1:1.250. Uma alta prevalência da variante cardíaca foi encontrada em Taiwan, onde é estimado afetar 1 em 1.600 machos. Manifestações clínicas em hemizigotos classicamente afetados que não possuem galactosidase detectável (LIN et al. 2009; MEHTA, 2002).

São poucos os estudos que avaliaram a prevalência de Doença de Fabry entre a população no Brasil. Em estudos realizados entre 2007 e 2008, envolvendo um pequeno número de pacientes, a prevalência variou de 0,36\% a 0,57\%.22-24.Já em um estudo mais recente, realizado no Estado da Bahia, que rastreou a Doença de Fabry entre 2583 pacientes do sexo masculino em HD, a taxa de prevalência de 0,12\% (YOGASUNDARAM et al., 2018; BOGGIO et al., 2009; MEHTA, 2002).

Os indivíduos analisados e diagnosticados com DLDs neste estudo, a Doença de Niemann-Pick tem um total 7,80\% da amostra, onde está distribuída em maior parte no Estado de São Paulo e em Pernambucocom maior número do sexo masculino. A doença deNiemann-Pick tem como característica principal, a deficiência de uma enzima lisossomal. A doença foi descrita pela primeira vez por Albert Niemann, em 1914, em uma criança com hepatoesplenomegalia, linfadenopatia e deterioração neurológica progressiva, que morreu antes dos 2 anos de idade. Os estudos histopatológicos foram posteriormente realizados por Ludwig Pick, demonstrando a presença de célulassemelhantes, mas não idênticas as encontradas na Doença de Gaucher (EMANUEL et al.2018;SIMONARO, 2002).

Em se tratando da comunidade judaica, dois estudos (Goldschmidt, 1960\& Frafir,1972) avaliaram a frequência de casamentos consanguíneos em Israel e encontraram altas frequências. Por conseguinte, as frequências são as praticamente mesmas quando se estudam judeus de diferentes países, como Alemanha, Polônia, Itália, França e Israel, segundo Wasserstein (2004), em 1934, levando em consideração a ascendência judaica da Europa Oriental, presente no início da vida. A pele pode ter uma pigmentação amarelo-amarronzada, os linfonodos estão aumentados e as manifestações oculares (mácula vermelho-cereja e opacificações da córnea) são evidentes. Poucos pacientes sobrevivem além dos 4 anos de idade. $\mathrm{O}$ tipo $\mathrm{B}$, ou variante não neuronopática (alélico com tipo A) assemelha-se ao tipo A, mas poupa o SNC (EMANUEL et al. 2018; ACUÑAet al, 2016; WASSERTEIN, 2004).

O termo NPD tipo E, foi usado no passado para designar pacientes com uma forma não neuronopática de início adulto da doença com hepatoesplenomegalia moderada,dos alelos mutantes em pacientes Judeus Asquenazes. Ao contrário do NPD tipo A, o NPD tipo B não ocorrem mais frequentemente na população judaica Ashkenazi, mais da metade dos pacientes relatados comNPD tipo B são de descendência turca, árabe ou norte-africana. Sendo responsável por quase $90 \%$ dos alelos mutantes em pacientes do tipo B do norte da Áfri- 
ca. Algum grau de correlação genótipo-fenótipo foi identificado. A prevalência de NPD tipos A e B combinado é cerca de 1 em 250.000 (FERREIRA, 2017; FREITAS et al., 2017; WASSERSTEIN, 2015).

Com Base dos dados deste estudo, a Doença de Pompe é mais prevalente em Rio de Janeiro (13,8\%), com um número maior no sexo feminino.Estima-se que possam existir no Brasil, de 1.000 a 3.500 pacientes com doença de Pompe.Apesar de ser uma doença pouco frequente, ela apresenta impacto significativo com alta morbidade de letalidade em sua forma infantil e alta morbidade em sua forma tardia (FERREIRA et al., 2016; HIRSCHHORN et al., 2011; MARTINIUK et al.,1998).

Os dados sobre a incidência da Doença de Pompe ainda são imprecisos, dada a raridade da doença. Dados dos Estados Unidos estimam que sua incidência global é de aproximadamente 1: 40.000.Estudos recentes que foram realizados em Taiwan e Áustria encontraram maior incidência de aproximadamente 1: 28.000. Na América Latina, apenas 88 pacientes foram informados a doença até 2012. Os dados do Brasil não estão disponíveis (CHIANGX, 2012; MECHTLER, 2012;MARTINIUK, 1998).

Com Base dos dados deste estudo, a Doença de MPS VI e MPS I é mais prevalente em São Paulo (6,38\%), em indivíduos do sexo feminino e sexo masculino.Lowry et al,1990 encontraram 28 casos de MPS com etiologia definida na Columbia Britânica (Canadá) em um período de 34 anos de investigação. Nesse período, nasceram vivas 1.298.469 crianças. Apenas um caso de MPSVI foi descrito e a maioria dos pacientes com MPS tinha origem no Norte da Europa. Um paciente possuía origem italiana e outra grega. Nenhum paciente de origem judaica foi encontrado. Nelson (1997),encontrou num período de 27 anos, na Irlanda do Norte, 34 casos de MPS. Nesse período, 839.517 crianças nasceram vivas. De acordo com estudos internacionais, a incidência do MPS varia de 1,9 a 4,5 / 100.000 nascimentos (ZELEI,
2018; PORONTHUIS et al.,1999).

A incidência real de MPS no Brasil é desconhecido. Em um estudo de Coelho e colaboradores(1997), relata o diagnóstico de erros inatos de metabolismo (EMI) em pacientes brasileiros de risco, as doenças de depósito lisossômico grupo frequente de IEM (59,8\% de todos os diagnósticos de IEM) e o MPS representou 54,5\% do LSD pacientes. A Rede MPS Brasil, uma iniciativa para melhorar o diagnóstico e a gestão do MPS doenças no Brasil, identificaram 161 novos pacientes com MPS entre abril de 2004 e setembro de 2006, conclui-se que a doença de MPS chegou até o Brasil através de imigrações e navegações, assim passando a doença de gerações para gerações de pais e filhos (FIGUEIRÊDO et al., 2018).

\section{CONCLUSÃO}

Com a ascensão do nazismo na Alemanha em 1933, a Alemanha experimentou uma nova onda de emigração. Boa parte desses refugiados era formada por judeus. Cerca de 16 mil conseguiram vistos de entrada para o Brasil, povoando grande parte do território brasileiro, passando os genes afetados de geração em geração.As etnias isoladas tendem a apresentar predisposição para distúrbios cromossômicos raros em outros grupos populacionais, Gaucher e Niemann-Pick são doenças comuns em certos grupos judaicos, embora jamais descritas em outros povos.

Portando, podemos concluir que a presença das doenças lisossômicas de depósito no Brasil tem relação direta com os conflitos políticos do passado. Pois, o rastreio genético dessas doenças coincide com a vinda dos judeus para o Brasil. Judeus Ashkenazim devem ser encaminhados à uma consulta de Aconselhamento Genético, poisa arte do aconselhamento repousa na determinação da melhor época e circunstância para o fornecimento de cada informação, que pode ser complexa. 


\section{REFERÊNCIAS}

ACUÑA,Mariana;MARTÍNEZ,Pablo.;MORAGA,Carolet al.Epidemiological, clinical and biochemical characterization of the $\mathrm{p}$. (Ala359Asp)SMPD1variant causing Niemann-Pick disease type B.Europe JornalHuman Genetic, v. 24, p.208-213, 2016. https://doi.org/10.1038/ejhg.2015.89

BARNESS,LewisA. et al. Clinicpathological conference: Oneyear-old infant with hepatosplenomegaly and developmental delay. American Journal of Medical Genetics.v. 28, n. 2, p. 411-431, 1987.

BERMAN,E. R.et al. Congenital corneal clouding with abnormal systemic storage bodies: A new variant of mucolipidosis. The Journal of Pediatrics.v. 84, n.4, p. 519-526, 1974.

BERNARDES, TaízaP. et al. Fabry disease: genetics, pathology, and treatment.Revista Associação Médica Brasileira. v.66, SUPPL 1, p.10-16, 2020.

BERNSTEIN, JorgeA. et al.Da busca pelo diagnóstico às incertezas do tratamento: desafios do cuidado para as doenças genéticas raras no Brasil. Ciência \& Saúde Coletiva. v. 24, n. 10 2019. Disponível em: https://doi.org/10.1590/1413812320182410.01612019.

BOGGIO,Paula et al. Doença de Fabry.Anais Brasileiros deDermatologia.v. 84, n.4, 2009.

BRADY,Roscoe O.;KANFER,JulianN.;MOCK,MichaelB.;FREDRICKSON,Donald S. The metabolism of sphingomyelin. II. Evidence of an enzymatic deficiency in Niemann-Pick disease. Proceedings of the National Academy of Sciences of the United States of America., v. 55, n. 2, p.366-369, 1966. doi:10.1073/pnas.55.2.366.

BREIGEIRON,Márcia K.;MORAES, VitóriaC.;COELHO,JaniceC. Sinais e sintomas na Doença de Gaucher: diagnósticos de enfermagem prioritários. Revista Brasileira de Enfermagem.v. 71, n. 1, p.113-20, 2018

CHAVES,RigobertoG.;CHAVES,MariliaF.;CAVALCANTE,Renata. G. Doença de Gaucher: revisão sistêmica e oftalmológica.Revista Brasileira de Oftalmologia. v. 4, p. 2, p. 43-50, 2018.

CHIANG,Shu-Chuanet al. Algorithm for Pompe disease newborn screening: results from the Taiwan screening program. Revista Molecular Genetics and Metabolism. 3(106): 281-6, 2012. Disponível em: < https: doi.org/10.1016/j.ymgme.2012.04.013 3> Acesso em: março. 2020.

COSTA,HugoA. P. et al. Doença de Gaucher e atuação do cirurgião-dentista: relato de caso. RevistaArchives ofHealthInvestigation. v. 7, n. 8, 2018. Disponívelem: http://dx.doi. org/10.21270/archi.v7i8.3033.
CROCKER,AllenC. The cerebral defect in Tay-Sachs disease and Niemann-Pick disease.Journal Neurochemistry.v. 7, p. 69-80, 1961.

EMANUEL,AnthonyI. et al. Small Bowel Mucosal Involvement and Mesenteric Mass Formation in a Young Female with Type 3 Gaucher Disease. A Case Report. Journal ofGastrointestinalandLiver Diseases.v. 4, p. 459-463, 2018.

EMENS, LeishaA.;DAVIS, S. Lindsey;OLIVER, Scott. C. N. et al. Association of Cancer Immunotherapy with Acute Macular Neuroretinopathy and Diffuse Retinal Venulitis. JAMA Ophthalmology.v. 137, p. 1, p. 96-100, 2019. doi:10.1001/jamaophthalmol.2018.5191

FERREIRA, CarlosR.;GAHL, William. A. Lysosomalstoragediseases. Translational Science of Rare Diseases, v. 2, n. 1-2, p. 1-71.2017.doi: 10.3233/TRD-160005.

FERREIRA,CamilaS. et al. Doença de Gaucher: uma desordem subdiagnosticada.RevistaPaulista dePediatria, v.1, n.291, p. 122-5, 2011.

FERREIRA,KarineM. S.;TORALLES,MariaBetaniaP.;MENDES, Carlos. MauricioC. Resposta ao uso da terapia de reposição enzimática na doença de Pompe: uma revisão sistemática com metanálises.Dissertação de Mestradoda Universidade Federal da Bahia, 2016.

FIGUEIRÊDO,BarbaraB. R. S. et al. Level of independence, functional capacity and respiratory muscle strength of patients with mucopolysaccharidosis type VI in the Northeast of Brazil. Revista BrasileiradeSaúdeMaternoInfantil.v.18, n. 1, p. 8392, 2018.

FREITAS, H elois a M. P.; MANÇAN O,A lexandre D.;RODRIGUES,RosanaS. et al. Niemann-Pick disease type B: HRCT assessment of pulmonary involvement.JournalBrasileiro dePneumologia. 2017; v. 43, n.6, p. 451-455. doi:10.1590/ S1806-37562017000000062.

HIRSCHHORN,Rochele;REUSER,ArnoldJ. J. Glycogen storage disease type II. Acid alpha-glucosidase (acid maltase) deficiency. In: Scriver C, Beaudet A, Sly W, Valle D, editors. The metabolic and mollecular bases of inherited disease. New York: Mac Graw-Hill, p. 3389-420, 2011.

KARAM,SimoneM.;SCHWARTZ,IdaV. D.; GIUGLIANI, Roberto. Introdução e aspectos clínicos. In: CarakushanskyG. Doenças genéticas em pediatria. Rio de Janeiro: Guanabara-Koogan, p. 155-8, 2001.

KHARROUBI,Melek;BEN FATMA,Lilia;RAIS,Lamia et al. Primary glomerulonephritis with predominant mesangial Immunoglobulin G deposits. La TunisieMedicale. 2018 Jul; v. 96, n. 7, p. 442-444. 
LIN,Hsiang-Yu;CHONG,Kah-Wai;HSU,Ju-Hui et al. High incidence of the cardiac variant of Fabry disease revealed by newborn screening in the Taiwan Chinese population. Circulation Cardiovascular Genetics. 2(5):450-456, 2009. doi:10.1161/CIRCGENETICS.109.862920.

MAHER, Paul. D.;HAFFNER,Marlene. E. Orphan drug designation and pharmacogenomics: options and opportunities. RevistaBiodrugs.v. 20, p. 71-79, 2006.

MARTINIUK, Frank et al. Frequency for glycogen storage disease type II in New York and estimates of affected individuals born with the disease. American Journal of Medical Genetics, v.1, n. 79, p. 69-72, 1998.

MECHTLER,ThomasP. et al. Neonatal screening for lysosomal storage disorders: feasibility and incidence from a nationwide study in Austria. Lancet.v. 379, p. 335-41, 2012.

MEHTA,Atul;HUGHES,Derralyn. A. Fabry Disease. In: R.A. Pagon, M.P. Adam, H.H. Ardinger, S.E. Wallace, A. Amemiya, L.J. Bean, et al., editors. RevistaGeneReviews(®), Seattle (WA) University of Washington, Seattle, 2002.

MOZAFARI, Hadi;KHATAMI, Shohreh;KIANI, Amir et al. Oxidative Stress Parameters, Trace Elements, and Lipid Profile in Iranian Patients with Gaucher Disease.Biological Trace Element Research.v. 193, p. 130-137, 2020. Disponívelem: https://doi.org/10.1007/s12011-019-01709-3.

PASTORES,GregoryM.;HUGHES,Derralyn. A. Gaucher Disease. In: Pagon RA, Adam MP, Ardinger HH, Wallace SE, Amemiya A, Bean LJ, et al., RevistaGeneReviews( $(\circledR)$, Seattle (WA) University of Washington, Seattle, 2018

POORTHUIS,B. J. H. M. et al. The frequency of lysosomal storage diseases in The Netherlands. Journal of Human Genetics.v. 105, p. 151-156, 1999.

PRADHAN,Dinesh;VARMA, Neelam;GAMI,Ashimita;HURA,Kanwaljeet. S.;MOHANTY,SambitK. Lysosomal storage disorders: Morphologic appraisal in Indian population. Journal of Cancer Research and Therapeutics. v. 13, p. 442-5, 2017.

SABATINI, DavidD., ADESNIK, Milton B. The Biogenesis of Membranes and Organelles. In: Valle DL, Beaudet AL, Vogel- stein B, Kinzler KW, Antonarakis SE, Ballabio A, et al., editors. The Online Metabolic and Molecular Bases of Inherited Disease [Internet]. New York, NY: The McGraw-Hill Companies, Inc.; 2014 [cited 2015 Jul 27]. Availablefrom: http://mhmedical com/content.aspx?aid=1102899807

SILVA, CassianoA.B. Doença de Fabry. Revista Médica UFPR.v.4, n. 1 , p. $23-30,2017$

SIMONARO, CalogeraM. et al. The demographics and distribution of type B Niemann-Pick disease: Novel mutations lead to new genotype/phenotype correlations.Journal of Human Genetics. v.71, n. 6, p. 1413-1419, 2002.

SOBREIRA,Elisa. A. P., BRUNEIRA, Paula. Evaluation of two years of treatment with enzyme replacement therapy in type 1 Gaucher disease patients of São Paulo State, Brazil. Revista BrasileiradeHematologiaeHemoterapia. v. 30, n. 3. São José do Rio Preto, 2008.

VANIER,M.T. et al. Deletion of arginine (608) in acid sphingomyelinase is the prevalent mutation among Niemann-Pick disease type B patients from northern Africa, Journal of Human Genetics.v. 92, n.4, p. 325-330, 1993.

WASSERSTEIN,MelissaP. et al. The natural history of type B Niemann-Pick disease: Results from a 10-year longitudinal study, RevistaPediatrics.v. 6, n.114, p. 672-677, 2004

YOGASUNDARAM,Haranet al. Elevated Inflammatory Plasma Biomarkers in Patients With Fabry Disease: A Critical Link to Heart Failure With Preserved Ejection Fraction.Journal America Heart Association.v. 21, 2018.

ZELEI,Tamás et al. Epidemiology of Sanfilippo syndrome: results of a systematic literature review. RevistaOrphanet Journal Rare Disease. v. 13, n. 53, 2018.

ZHOU, Chi et all. Identification of a novel loss-of-function mutation of the GLA gene in a Chinese Han family with Fabry disease.RevistaBMC Medical Genetics. v. 1, n.19, p. 219, 2018.

ZIMRAN,Ari et al. Long-term efficacy and safety results of taliglucerase alfa through 5 yearsin adult treatment-naïve patients with Gaucher diseaseBlood Cells. Blood Cells, Molecules, and Diseases. v. 78(set), p. 14-21, 2019. 